
ПРОЦЕССЕ С ПРИМЕНЕНИЕМ ВИРТУАЛЬНОЙ КОМПЬЮТЕРНОЙ ЛАБОРАТОРИИ

\author{
Сидоров Дмитрий Сергеевич ${ }^{1}$, Белов Михаил Александрович ${ }^{2}$
}

\author{
${ }^{1}$ Студент; \\ ГБОУ ВО МО «Университет «Дубна», \\ Институт системного анализа и управления; \\ 141980, Московская обл., г. Дубна, ул. Университетская, 19; \\ e-mail: dimasid@uni-dubna.ru. \\ ${ }^{2}$ Кандидат технических наук, дочент; \\ ГБОУ ВО МО «Университет «Дубна», \\ Институт системного анализа и управления; \\ 141980, Московская обл., г. Дубна, ул. Университетская, 19; \\ e-mail: belov@uni-dubna.ru.
}

В статье описывается наглядный пример типовых аппаратно-программных комплексов, который учашчеся могут самостоятельно проектировать и создавать с применением средств виртуализации и контейнеризации в среде виртуальной компьютерной лаборатории (ВКЛ). Данная статья позволит более точно представлять условия и ограничения, которые приходится преодолевать в традиционных компьютерных классах, а также сформировать требования к специализированному контуру ВКЛ, который позволит учащимся отправлять запросы на конфигурирование оборудования, инвентаризировать оборудование, реализовать систему интеллектуального управления очередью и ресурсами, а также проверять правильность коммутации оборудования на основе машинного обучения с подкреплением. Обучение основам проектирования IoT-решений на Arduino u Raspberry Pi и создание управляющего программного обеспечения, позволяет готовить специалистов, способных сделать вклад в создание $и$ развитие высокотехнологичных перспективных программнотехнологических решений.

Ключевые слова: виртуальная компьютерная лаборатория, IoT, удаленное обучение, комплекс по мониторингу авиатрафика, лаборатории в учебном процессе, новые методы обучения, новые технологии обучения, виртуализация, контейнеры, новые стандарты обучения, обучение в условиях коронавируса, коронавирус.

\title{
DESIGN OF HARDWARE-SOFTWARE SYSTEMS IN THE EDUCATIONAL PROCESS WITH THE USE OF VIRTUAL COMPUTER LAB
}

\section{Sidorov Dmitry ${ }^{1}$, Belov Mikhail ${ }^{2}$}

\author{
${ }^{\text {IStudent; }}$ \\ Dubna State University, \\ Institute of system analysis and management; \\ 141980, Dubna, Moscow region., Universitetskaya str., 19; \\ e-mail:dimasid@uni-dubna.ru. \\ ${ }^{2}$ Candidate of Science in Engineering, associate professor. \\ Dubna State University, \\ Institute of the system analysis and management. \\ 141980, Moscow region, Dubna, st. Universitetskaya, 19; \\ e-mail:mbelov@live.ru.
}

The article describes an illustrative example of IoT-solutions that students can independently design and create using virtualization and containerization tools in a Virtual Computer Lab (VCL) environment. This article will allow you to more accurately represent the conditions and limitations that have to be overcome in traditional computer classes, as well as design requirements for a special VCL circuit, which will allow students to send requests for equipment configuration, inventory equipment, implement an intelligent queue and resource management system, and also verify correct connections of equipment based on machine 
learning with reinforcement. Learning the basics of designing IoT-solutions on Arduino and Raspberry Pi and creating management software allows you to train specialists who can contribute to the creation and development of high-tech advanced software and technology solutions.

Keywords: virtual computer laboratory, IoT, distance learning, e-learning, LMS, Arduino, Raspberry Pi, system for monitoring air traffic, laboratories in the educational process, new teaching methods, new teaching technologies, new educational standards, training in coronavirus, coronavirus, virtual computer lab, virtualization, containers.

\section{Введение}

На сегодняшний день происходит развитие интернета вещей, поэтому при подготовке ИТспециалистов существует потребность в разработке автономных аппаратно-программных комплексов и взаимодействия с ними. Под взаимодействием понимается, возможность удалённой коммутации компонентов на основе технологий расширенной реальности для комплектации сборки комплекса с экспертной системой проверки.

Интернет вещей (Internet of Things, IoT) - новая стадия развития интернета, когда к нему подключено больше вещей, чем людей. Переход к ней случился в 2008-2009 годах, когда количество устройств в сети обогнало численность населения Земли. $I o T-$ не одна, а целый стек технологий. Это и создание датчиков, и множество протоколов их взаимодействия. Объекты могут общаться между собой через Wi-Fi, Bluetooth, LPWAN, BLE, Ethernet, RFID, ZigBee и другие виды беспроводной связи. Частным случаем интернета вещей является межмашинное взаимодействие.

Разработка мультимедийных учебно-научных лабораторий и их использование в инженерном образовании являются перспективным направлением в обучении современным высоким технологиям, подготовке высококвалифицированных научных кадров и отраслевых специалистов, а также повышении квалификации инженерно-технических работников и сотрудников предприятий промышленного сектора.

В статье подробно описывается система мониторинга авиатрафика, которую можно собрать из подручных средств, на примере которой выполняется совершенствование Виртуальной Компьютерной Лаборатории для обеспечения возможности проводить опыты без непосредственного контакта с реальной установкой или при полном отсутствии таковой, а также предоставления сервиса удалённого монтажа оборудования с возможностью просмотра видео в формате 360 градусов (панорамное видео).

\section{Виртуальная компьютерная лаборатория}

Виртуальная компьютерная лаборатория (ВКЛ), созданная в Институте системного анализа и управления государственного университета «Дубна» доцентом Беловым М.А. в 2007, успешно совершенствуется и развивается, а в условиях цифровой трансформации становится незаменимым инструментом подготовки квалифицированных ИТ-специалистов, профили компетенций которых соответствуют современным потребностям рынка труда. ВКЛ предоставляет собой набор аппаратных и программных средств виртуализации и контейнеризации, которые обеспечивают гибкое предоставление и использование вычислительных ресурсов в форме облачных Интернет-услуг по требованию с интегрированной системой управления знаниями. Система управления знаниями и принципы самоорганизации, заложенные в основу ВКЛ позволяют сформировать однородную образовательную среду с элементами когнитивного представления внутренних операционных ресурсов на основе визуальных моделей и частичной автоматизации основных технологических операций с применением экспертной системы, что снижает порог вхождения и позволяет значительно быстрее получить результат в виде функционирующей многокомпонентной информационной системы. По сути, теперь учащимся не нужно решать разрозненные, порой оторванные от реальной практики задачи, ведь им предоставляется прекрасная возможность самостоятельно развёртывать и изучать современные информационные системы и самые последние достижения информационных технологий на основе актуальных методологий, теорем и алгоритмов, для создания перспективных программнотехнологических решений в области цифровой трансформации и ИТ-сервисов массового обслуживания. Компонентами архитектуры ВКЛ являются [3-7]: 
- Интегрированная система управления ВКЛ, объединяющая в себе различные сервисы и подсистемы.

- Сервер повышенной надежности - центр обработки данных (ЦОД) лезвийной архитектуры с возможностью резервирования компонентов.

- Коммуникационные сетевые магистрали.

- Система резервного копирования.

- Гипервизоры. Виртуальные машины. Контейнеры.

- Система интеграции с каталогом пользователей, подсистема групповых политик.

- Интернет-ориентированная система управления аппаратным и программным обеспечением, а также источниками бесперебойного питания.

- Экспертная система формирования конфигурации виртуальных машин и сетевой инфраструктуры.

- Система управления знания, интегрированная с системой онлайн коллаборации.

- Специализированные предметно-ориентированные модули, подсистемы, для обеспечения возможности работы с междисциплинарными программно-технологическими решениями.

\section{Задачи виртуальной компьютерной лаборатории}

1. Освоение сложного корпоративного и иного программного обеспечения, выполнение всего цикла задач создания виртуальных серверов.

2. Развертывание и настройка программного обеспечения, а также его использования на проблемно-ориентированных практических примерах, позволяющих формировать профессиональные компетенции и развивать конструктивное, аналитическое и системное мышление учащихся.

3. Выполнение практических заданий в рамках семинарских занятий.

4. Предоставление возможности удаленного использования корпоративного программного обеспечения и иного программного обеспечения, используемого в учебном процессе, для самоподготовки.

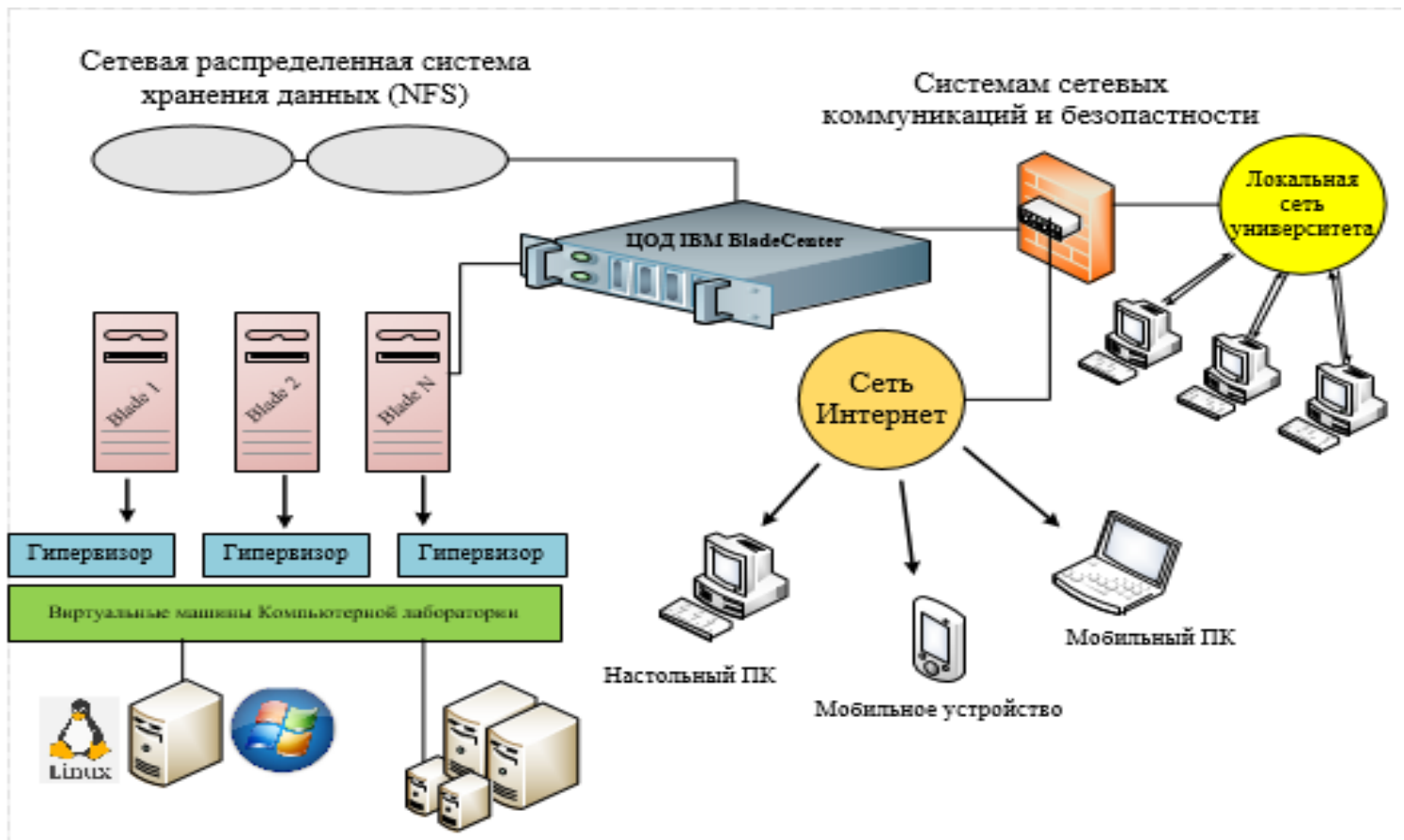

Рис. 1. Архитектура виртуальной компьютерной лаборатории 


\section{Интегрированная система управления виртуальной компьютерной лабораторией}

Интегрированная система управления обеспечивает взаимосвязь перечисленных компонентов, предоставляя единый, централизованный интерфейс для доступа ко всем функциональным частям и компонентам ВКЛ через браузер. Эта система определяет возможности централизованного управления, мониторинга и удаленной работы. За счет семантической разметки страниц она позволяет с какой-либо открытой веб-страницы перейти на связанные с ней страницы, содержащие дополнительные или родственные сведения, а также настройки, необходимые для удобной, продуктивной и интуитивно-понятной работы в системе [1].

Интегрированная система управления ВКЛ предоставляет удаленный доступ ко всем подсистемам, в том числе и аппаратным, позволяя администраторам лаборатории удаленно обновлять микропрограммы всех ее компонентов и позволяет так же удаленно проводить диагностику аппаратных частей ВКЛ, перезагружать их или даже выключать, что обеспечивает мобильность в управлении и обслуживании всей аппаратной платформы виртуальной компьютерной лаборатории [2].

Центральный доступ к программной платформе позволяет загружать и редактировать дистрибутивы программного обеспечения, операционных систем, интерактивных учебников, проводить контроль качества знаний, организовывать вебинары, контролировать усвояемость и успеваемость, формировать индивидуальные траектории обучения.

\section{Контроллеры интернета вещей}

Современная база электронных компонент и измерительная техника предоставляет весьма широкие возможности для экспериментатора и позволяет создавать измерительные комплексы, обеспечивающие не только сбор данных, но и организацию обратной связи для управления экспериментальным макетом. В то же время для создания специализированного измерительного комплекса необходимы большие временные вложения, а также высокая инженерно-техническая квалификация разработчиков.

С другой стороны, универсальные платформы, предлагающие готовые мощные аппаратные модули сбора данных и управления, работающие в широком диапазоне частот и амплитуд сигналов, обеспечивающие их анализ и обработку, а также управление оборудованием с помощью легких в освоении языков графического программирования, обладают при решении ряда задач недостаточной гибкостью. Они отличаются высокой ценой и часто требуют выделения значительных рабочих площадей. В такой ситуации определенную тактическую нишу занимает аппаратно-программная платформа Arduino. Это инструмент для проектирования электронных устройств, более плотно взаимодействующих с окружающей физической средой, чем стандартные персональные компьютеры. Платформа, предназначенная для «physical computing» с открытым программным кодом, построенная на простой печатной плате с современной средой для написания программного обеспечения. Применяется для создания электронных устройств с возможностью приема сигналов от датчиков температуры, давления, влажности, освещенности, измерения расстояния, компаса, GPS и ГЛОНАС навигаторов, так же имеется возможность управлять платформой через все виды беспроводных сетей, которые могут быть подключены к нему.

Данная платформа для образовательных учреждений позволяет получить возможность, развить навыки программирования на практике, а также освоить азы схемотехники. Учащийся может использовать готовые платы расширения или просто напрямую подключить к плате все необходимые элементы. Остальные усилия будут направлены на разработку и отладку управляющей программы на языке высокого уровня.

В итоге доступ к разработке микропроцессорных устройств получили не только профессионалы, но и все желающие вступить на путь инженерного творчества. Наличие готовых модулей и библиотек программ позволит новичку в электронике создавать готовые работающие устройства для решения не только своих задач, но и общественных задач. Варианты использования ограничены только возможностями микроконтроллера и имеющегося варианта. 
Её отличает низкая цена, наличие бесплатного программного обеспечения, возможность быстрого освоения за счет использования упрощенных языков программирования, минимальные требования к наличию дополнительных элементов и монтажного оборудования. При этом платформа предоставляет широкие возможности по генерации сигналов, сбору данных и управлению ходом эксперимента. Само программное обеспечение может быть модифицировано опытными пользователями, поскольку весь проект изначально предполагает открытую архитектуру с возможностью расширения.

Raspberry Pi - это серия миниатюрных одноплатных компьютеров размером с банковскую карту. Разработчиком является The Raspberry Pi Foundation - благотворительная организация, основанная в 2009 году для содействия изучению базовой информатики в школах. Проект создавался как образовательный, отлично подходящий для изучения основ электроники, обучения информатике, но впоследствии получила намного более широкое применение и популярность, чем ожидали его авторы. Работает в основном на операционных системах, основанных на Linux ядре. Так же возможна установка Windows 10 IoT. Большая часть моделей, распространяется полностью собранным на четырёхслойной печатной плате. В стандартной комплект поставки входит только сам мини-компьютер. Корпус, блок питания и карту памяти необходимо заказывать отдельно [9-12].

В целом представляет собой полноценный компьютер и, следовательно, предлагает большую вычислительную мощность по сравнению с Arduino. В результате он лучше всего используется для реализации таких задач, которые будут вызывать у Arduino сложности, например, обработка данных и визуализация.

\section{Пример аппаратно-программного комплекса на Raspberry Pi для мони- торинга авиатрафика}

В рамках магистерской работы, Сидоровым Д.С. был собран тестовый комплекс, состоящий из микрокомпьютера Raspberry Pi 4, RTL-SDR приемника, фильтра (блокирующий вещание $F M$ станций диапазона 88 - 108 МГЦ) и телескопической антенны. Комплекс работает под управлением операционной системы Raspbian (основанная на Debian) для Raspberyy Pi, а с помощью программного обеспечения - RTL1090 и SDRsharp выполняется приём радиосигнала воздушных судов (в широком диапазоне). К комплексу можно подключиться удаленно и получать реальные данные.

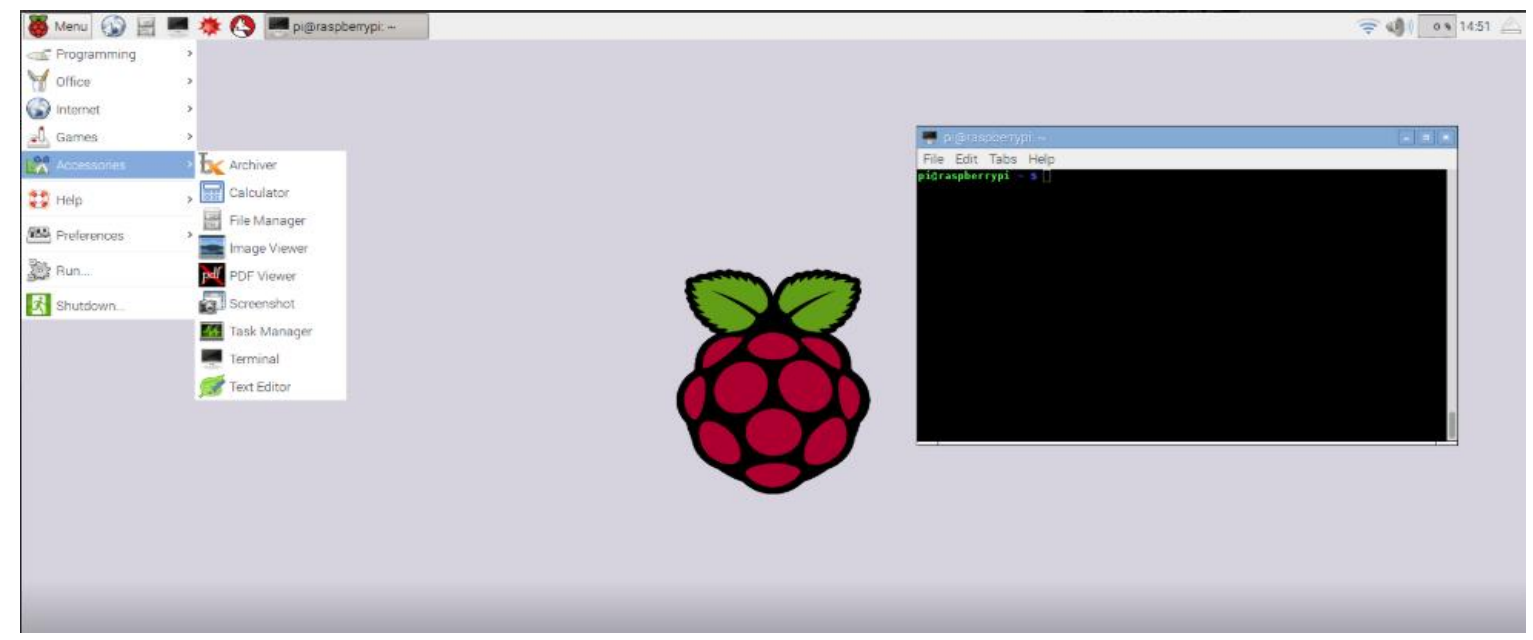

Puc. 2. Операционная система Rasbian 


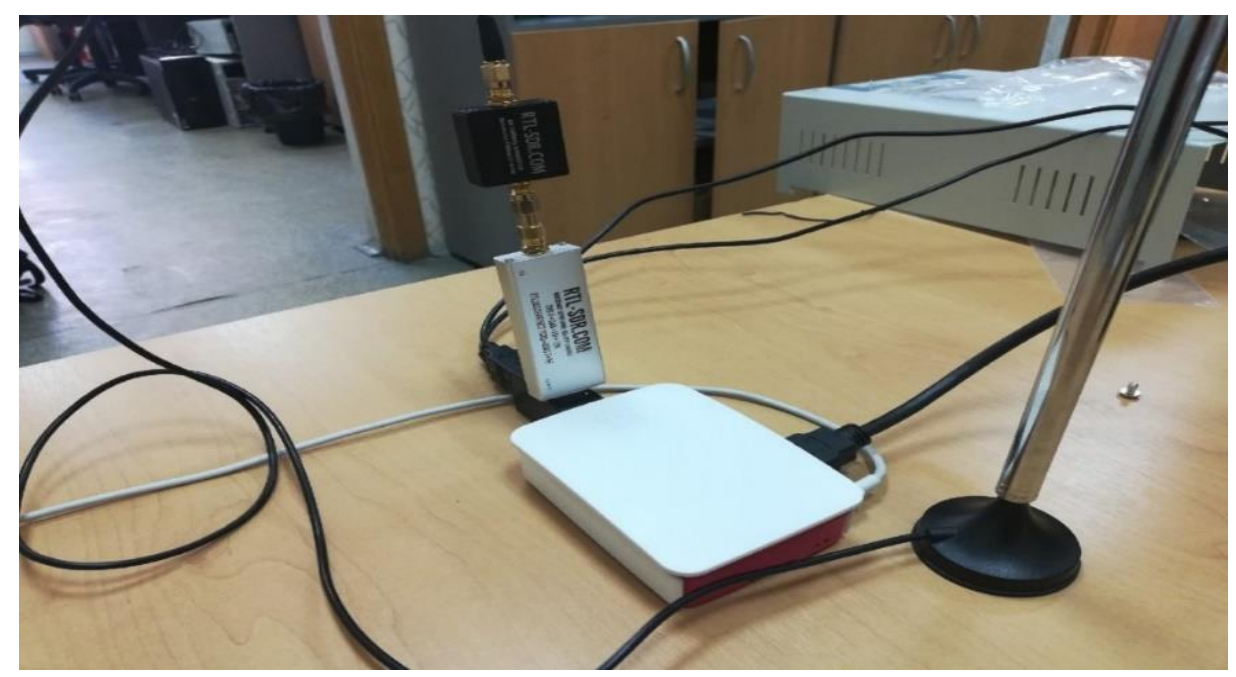

Рис. 3. Тестовый комплекс в сборе

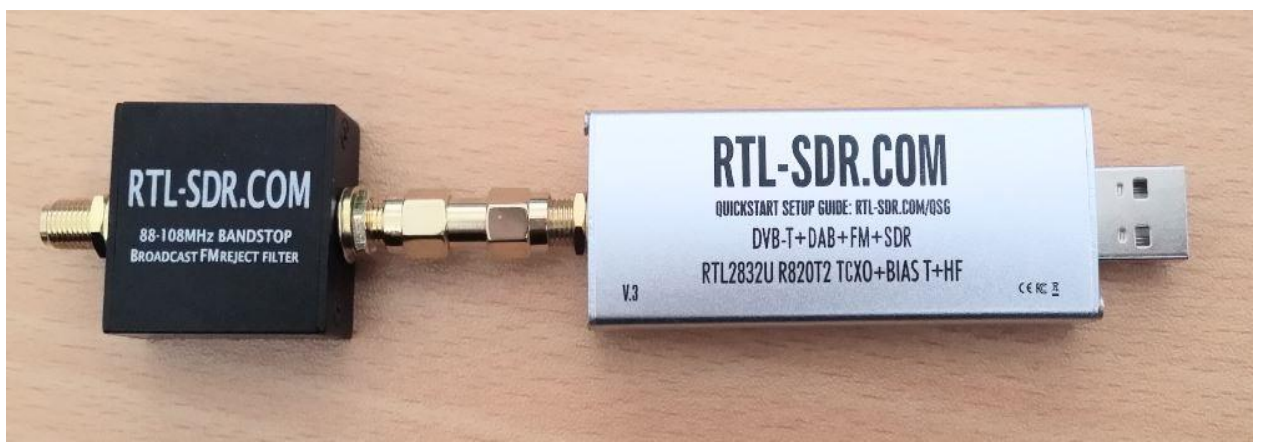

Puc. 4. RLT-SDR приемник с фильтром

\section{Описание элементов управления и настроек SDRSharp}

На рис. 5 представлено окно главное программы, запущенной на виртуальной машине, связанной c $R T L$ приемником, настройки которого приведены на рис. 6.

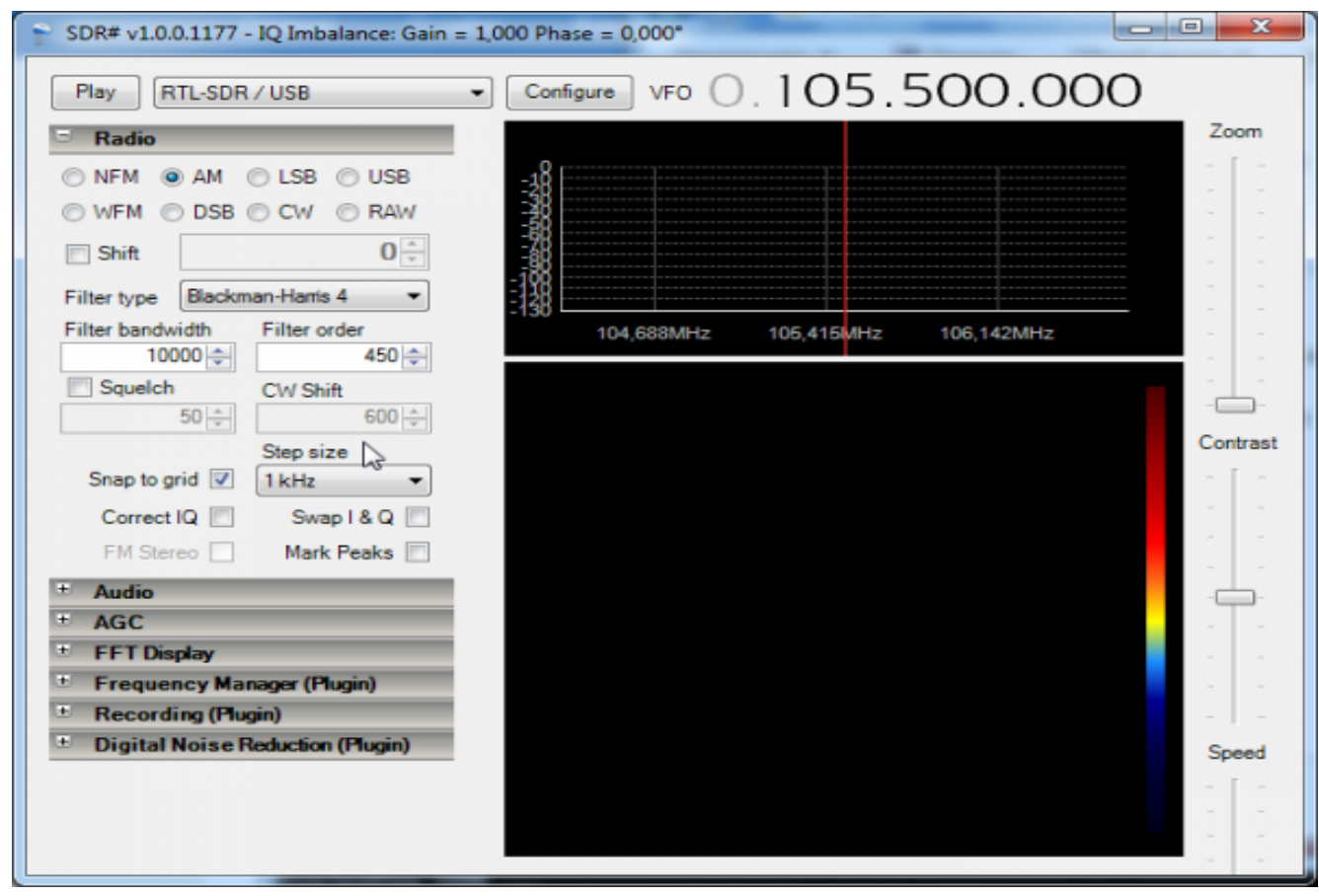

Puc. 5. 3anyck SDRSharp 


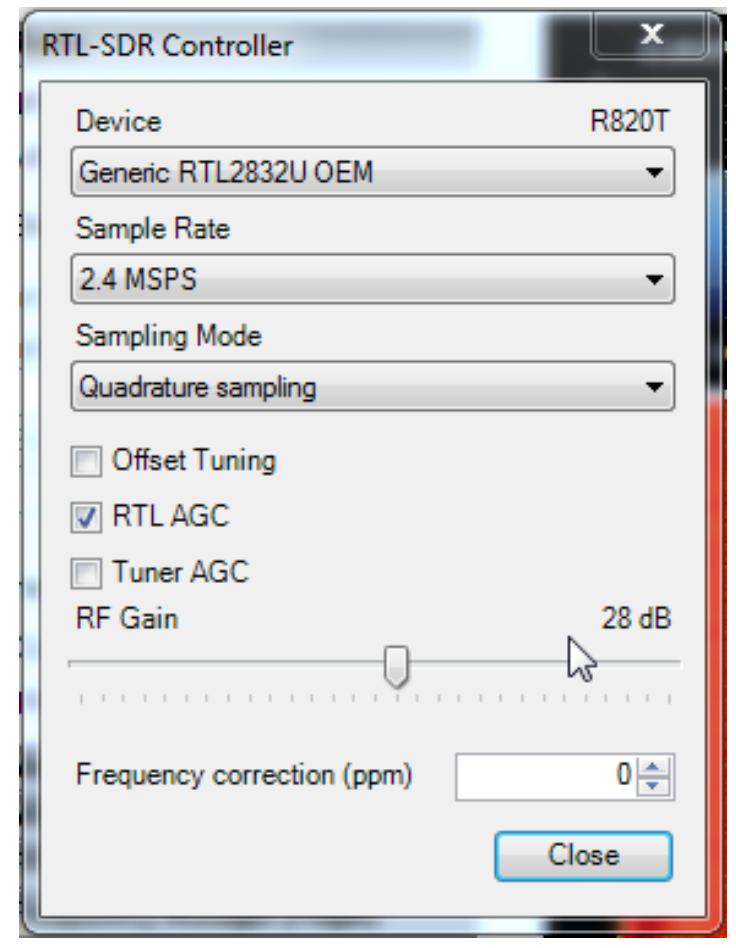

Рис. 6. Настройка приемника

Учащийся удалённо может настроить следующие параметры:

[Sample Rate] - (Частота дискретизации RTL) Ширина полосы приемника, 2048 MSPS это 2.048 МГц. Полосу можно менять от 0,25 МГц до 3,2 МГц. Чем больше полоса, тем больше нагрузка на процессор, в условиях виртуализации, было эмпирически подобрано значение 2.4 MSPS.

[Sampling Mode] - режим работы RTL устройства. Для работы необходим режим квадратурного приема. Он установлен по умолчанию. Есть ещё режимы оцифровки $I$ канала или $Q$ канала. Это специфичные режимы и в обычных условиях надобности в них нет.

[Offset Tuning] - это полезная опция при использовании тюнера E4000. Переключает режим работы входа $R T L$ с нулевой частоты на промежуточную не нулевую. Установка этой опции позволяет полностью избавится от «палки» посередине экрана.

[RTL AGC]- Автоматическая регулировка усиления на участке «Смеситель тюнера - АЦП $R T L 2832 »$, этот параметр требуется установить перед началом работы.

[Tuner AGC] - Автоматическая регулировка усиления на участке «Вход приемника - МШУ (малошумящий усилитель) - Смеситель». Данная АРУ (автоматическая регулировка усиления) работает не очень хорошо, многое зависит от антенны, условий приема и диапазона.

[RFGain] - Ручная регулировка усиления тюнера. Позволяет самостоятельно менять усиление входного тракта тюнера при отключенной [Tuner AGC]. Для первого запуска, имеет смысл установить этот регулятор в районе 25 - 36 дБ.

[Frequency correction PPM] - Коррекция частоты опорного генератора тюнера.

На рис. 7 показана модуляция сигнала, и для обеспечения нормальной работы системы потребуется установить опцию Correct IQ во вкладке Radio, для лучшего подавления зеркального канала и опцию Filter Audio во вкладке Audio (в левой части главного экрана на рис. 8), которая уберёт высокочастотный шум и треск. 


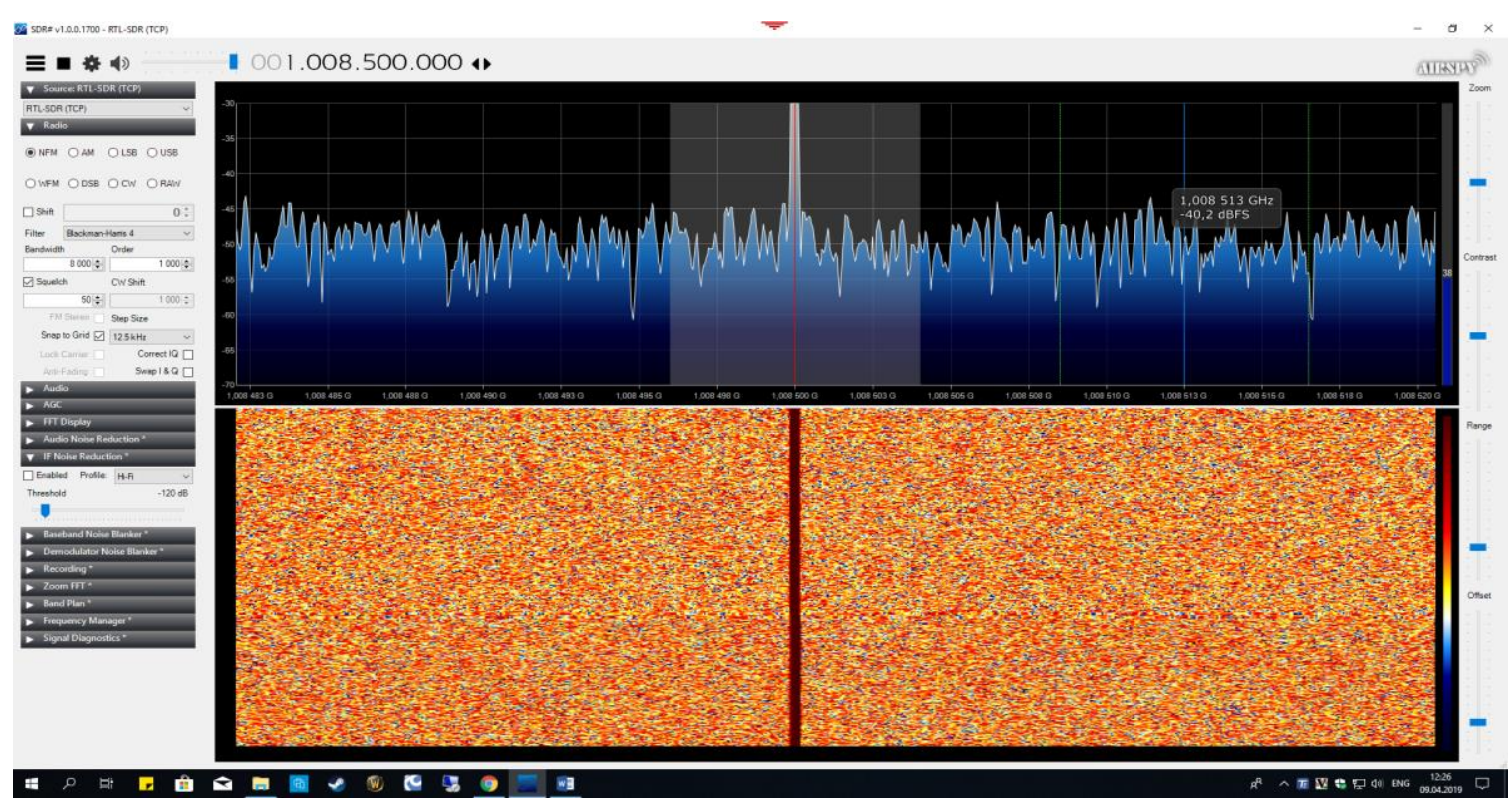

Рис. 7. Модулячия сигнала

Также на рис. 8 располагаются основные элементы системы управления аппаратно-программным комплексом по мониторингу авиатрафика.

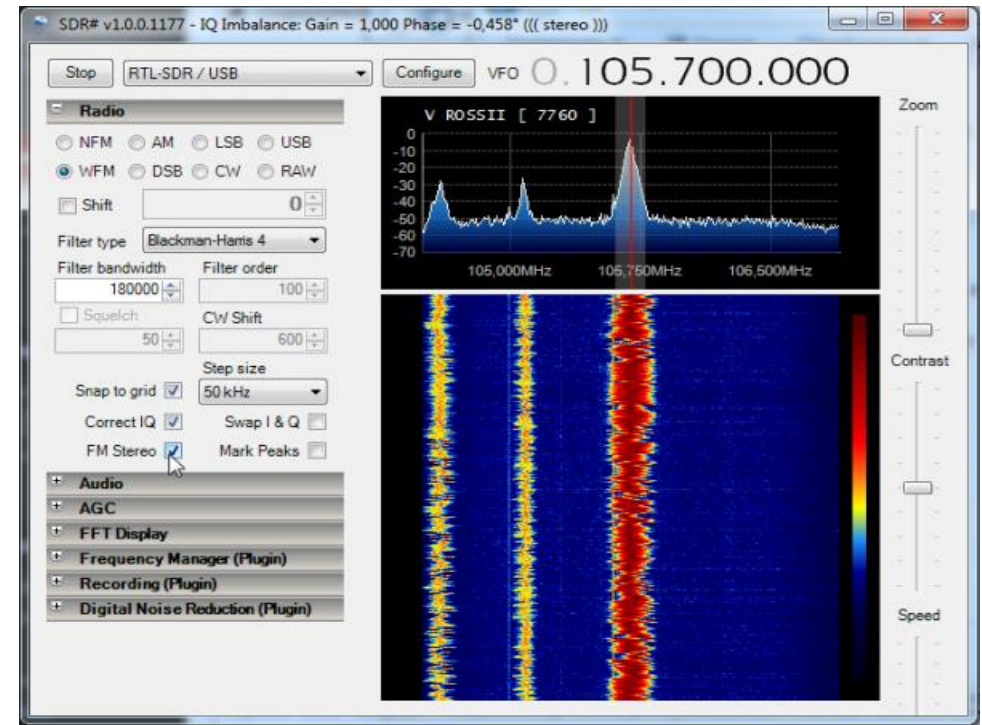

Рис. 8. Главный экран

Для наглядности, рассмотрим основные из них:

[STOP / PLAY] - кнопка запуска / остановки приема.

[CONFIGURE] - вход в настройки источника сигнала.

[VFO] - отображение частоты приёма. Мышкой, курсорными или цифровыми клавишами можно менять частоту приёма непосредственно в этом поле.

- ползунок [Zoom] - изменение масштаба отображения анализатора спектра и водопада;

- ползунок [Contrast] - смещение палитры водопада;

- ползунок [Speed] - изменение частоты обновления водопада и анализатора спектра.

Черное окно вверху - анализатор спектра принимаемой полосы частот. В этом окне кроме индикации спектра с помощью мышки возможно изменять частоту настройки.

Черное окно внизу - водопад. Также позволяет изменять частоту приема при помощи мышки. 


\section{Панель элементов управления}

Для обеспечения работоспособности аппаратно-программного комплекса, с помощью панели управления на рис. 9 были сделаны необходимые настройки параметров, показанных на этом же рисунке, а ниже мы приводим более детальное описание каждого их них.

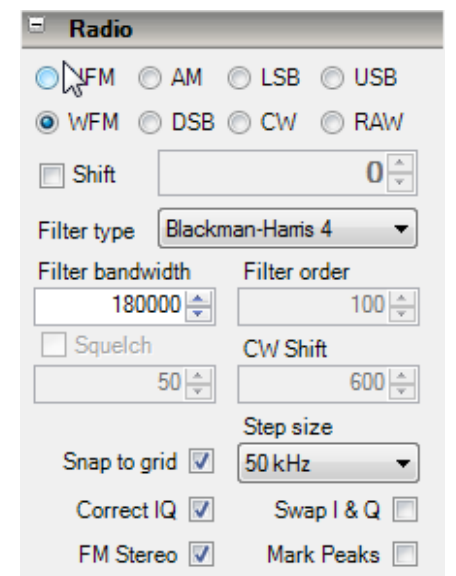

Рис. 9. Панель управления - Radio

[NFM AM LSB USB WFM DSB CW RAW] - переключение видов модуляции принимаемого сигнала. $R A W-$ прием без демодуляции сигнала.

[Shift] - смещение индикации частоты приема. Галочка включает, а цифровое поле задаёт смещение. Используется в основном с конвертерами для индикации точной частоты приёма.

[Filter type]- выбор оконного фильтра. Относится к теории цифровой обработки сигналов. Практической пользы от смены фильтров в процессе экспериментов не было замечено.

[Filter bandwidth] - полоса фильтра принимаемого сигнала.

[Filter order] - добротность фильтра. Влияет практически на все фильтры в программе, пропускающие принимаемый сигнал.

[Squelch] - шумоподавитель. Данная опция позволяет выполнять настройку чувствительности.

[Snap to grid] - привязка изменения частоты к сетке с шагом, указанным в цифровом поле [Step size].

[Correct IQ] - автокоррекция дисбаланса фазы и уровня $I Q$ сигнала. [Swap I \& $Q$ ] - меняет местами $I$ u $Q$ составляющие сигнала. Для $R T L$ устройств функция не используется.

[FM stereo] - стереодекодер для WFM приема.

Управление звуком вынесено на отдельную панель, приведённую на рис.10.

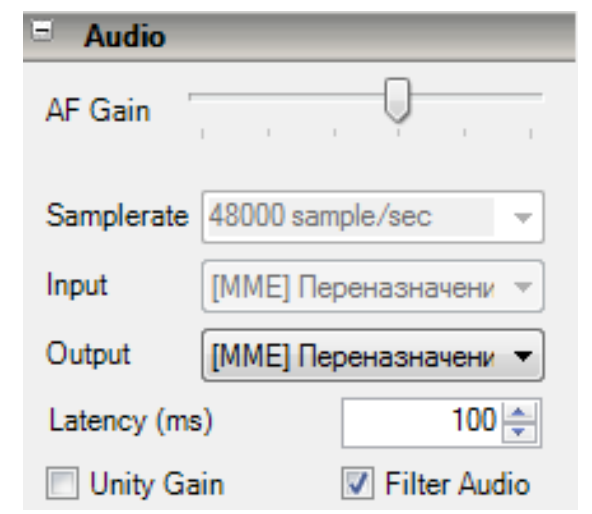

Рис. 10. Панель управления - Audio 
С помощью данной панели имеется возможность выполнять следующие формулировки:

[AF Gain] - громкость.

[Samplerate] - частота дискретизации звукового потока на выходе.

[Input] - выбор звукового входа.

[Output] - выбор устройства для воспроизведения звука.

[Latency] - Размер буфера накопления сигнала в миллисекундах.

[Filter Audio] - включение звукового фильтра на выходе. Фильтр не настраиваемый.

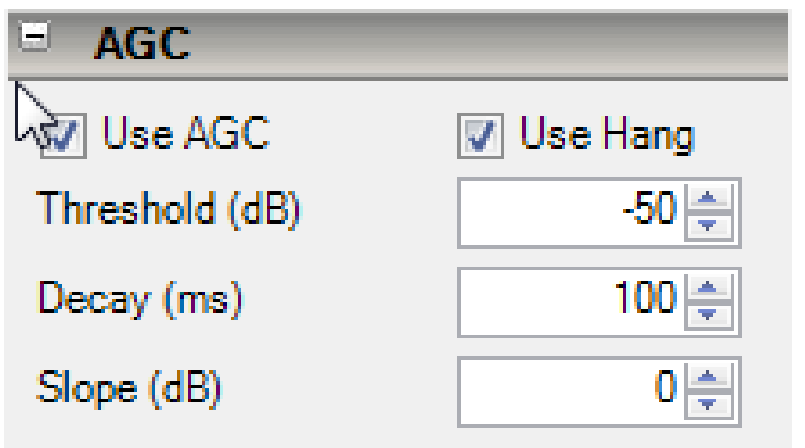

Рис. 11. АGC-Автоматическая регулировка усиления

Надо отметить, что для приемного тракта существует несколько автоматических регулировок усиления. В нашем случае $A G C$ на рис. 11 является программной, и призвана поддерживать уровень звука после демодуляции на одном заданном уровне и не влияет на общее усиление тракта от антенны до демодуляции.

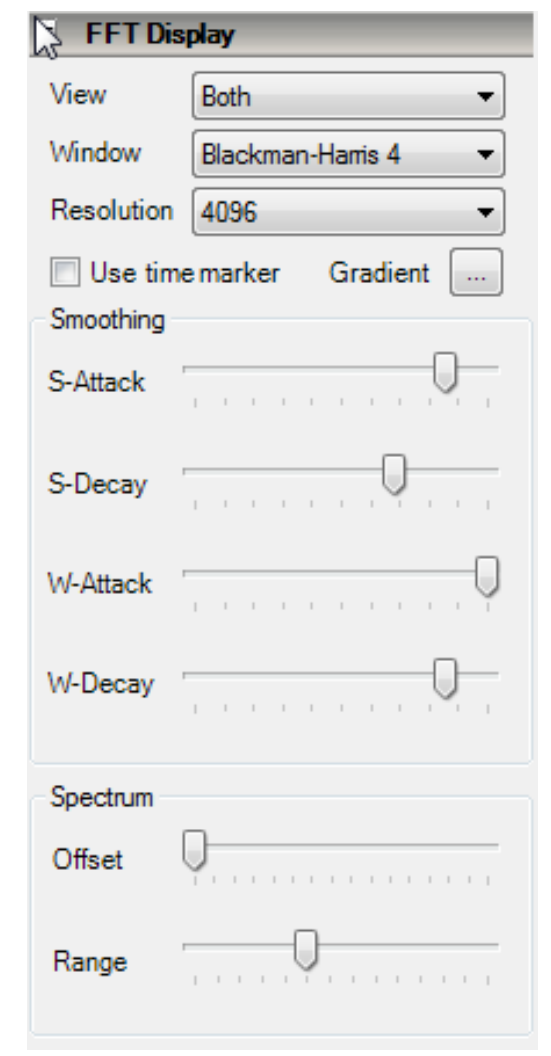

Рис. 12. Панель управления - FTP Display

Также определённый интерес может представлять настройка опций анализатора частотного диапазона. В процессе экспериментальной деятельности учащиеся могут менять следующие настройки: 
[View] - выбор отображения анализатора спектра, водопада или обоих одновременно.

[Window] - выбор оконной функции для анализатора и водопада. Из теории цифровой обработки сигналов. Оказывает некоторое влияние на отображение.

[Resolution] - количество точек преобразования фурье входного $I Q$ сигнала. Чем больше точек, тем чётче картинка спектра и больше нагрузка на процессор.

[Use time marker] - вывод на водопад информации о времени и дате.

[Offset] - смещение максимума анализатора спектра.

[Range] - (диапазон) смещение минимума анализатора спектра.

\section{Программное обеспечение RTL1090 для приемника RTL-SDR}

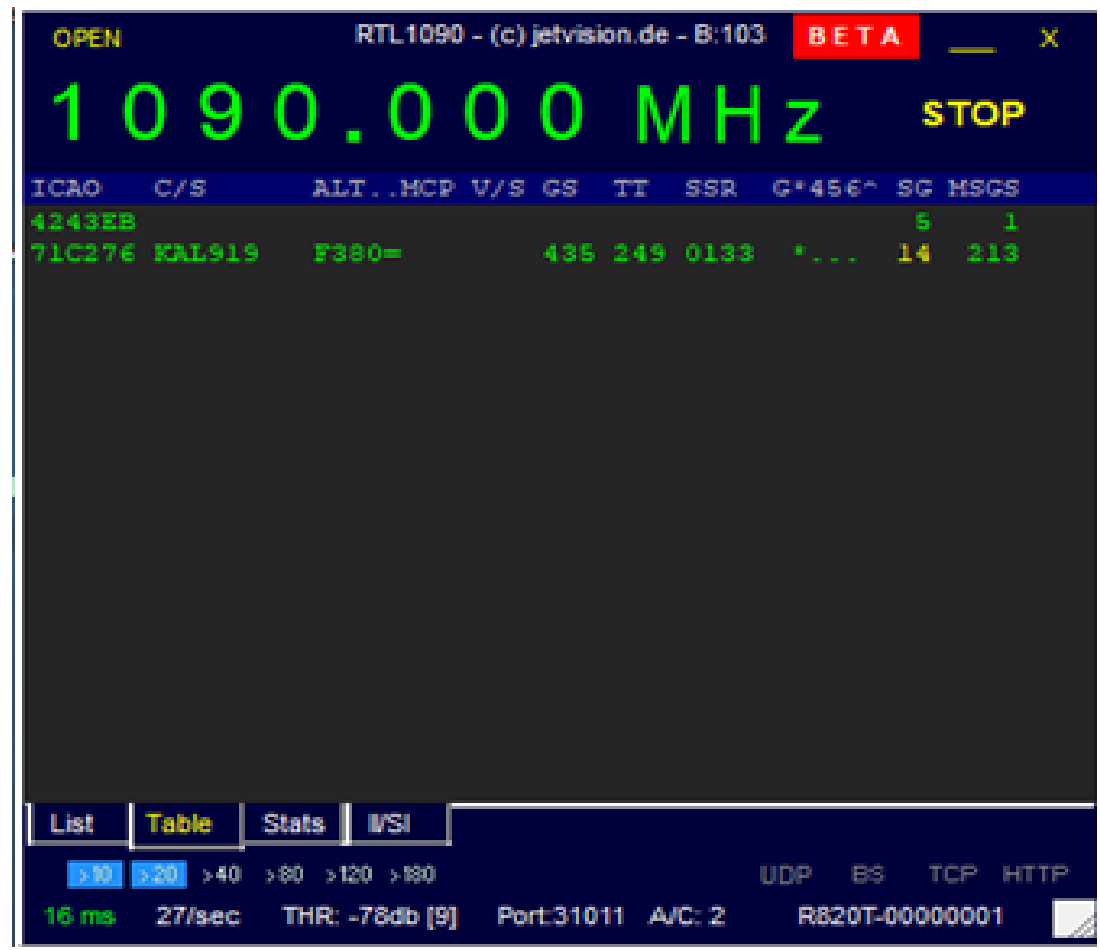

Рuc. 13. Приложения RTL1090

Приложение RTL1090 предназначено для совместного использования с программами Planeplotter, flightradar24, Virtual Server, ADS-Scope, Globe-S RTL и других программ с поддержкой $A D S-B$. Программа является приемником и детектором кадров $A D S-B$ идентификаторов / RFID / транспордеров самолетов.

К особенностям работы с программой относятся рекомендации:

- не устанавливать в каталог C:VProgram Files из-за проблем с правами администратора; - использовать настройки: Tuner $A G C$ - ON, RTL AGC - ON;

Программа успешно принимает данные и в условиях плотной городской застройки, и в условиях сельской глубинки, что объясняется высотой передатчика самолета, прямолинейным распространением волн и отсутствием в воздухе значительных помех, мешающих прохождению радиосигнала. На рис. 14 легко увидеть, что собранный из подручных средств комплекс, интегрированный в среду ВКЛ, позволяется получать результаты, релевантные тем, что получены из системы FlightRadar 24.

Вывод: виртуальная компьютерная лаборатория позволяет создавать аппаратно-программные комплексы, аналогичные рассмотренному в данной статье, однако для нормального функционирования требуются лаборанты и приобретение достаточно масштабного перечня устройств, необходимых для обеспечения одновременной работы всех вовлечённых и заинтересованных учащихся. 


\section{Опыт использования виртуальной компьютерной лаборатории в условиях коронавируса}

Одной из основных отличительных черт виртуальной компьютерной лаборатории являются принципы самоорганизации, которые позволяют студентам перейти от жесткой системы групповых политик безопасности компьютерного класса к системе без ограничений прав и свобод внутри неё, что должно способствовать развитию чувства личной ответственности, уважения к коллегам и толерантности, что должно обеспечить прочную основу для укрепления и развития основных культурных ценностей в образовательной среде.

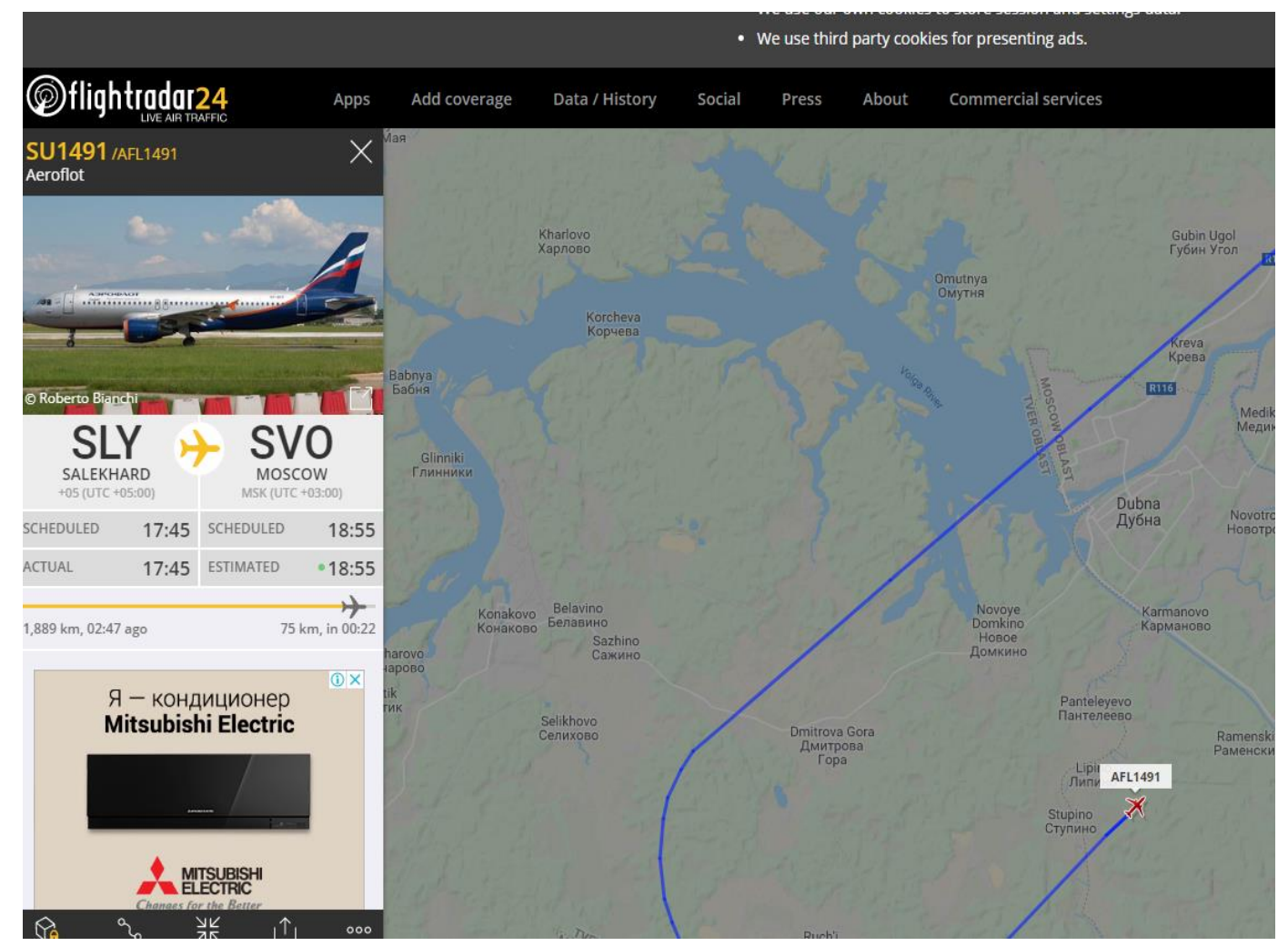

Рис. 14. Оиенка работы созданного аппаратно-программного комплекса в среде виртуальной компьютерной лаборатории и системь Flightradar24

Однако, условия коронавируса, вынудили нас использовать виртуальную компьютерную лабораторию для реализации практических заданий не только связанных с применением многокомпонентных информационных систем, но и для проектирования и создания аппаратно-программных комплексов.

Текущее состояние ВКЛ, позволяет реализовывать комплексы, подобные тому, что описан в этой статье, исключительно при непосредственном нахождении лаборанта и ручной коммутации оборудования и его подключения к соответствующим серверам ВКЛ. Вместе с тем, проведенный эксперимент позволил сформировать требования к специализированному контуру ВКЛ, который призван автоматизировать отправку запросов на конфигурирование оборудования, инвентаризацию оборудование, реализацию системы интеллектуального управления очередью и ресурсами, а также проверку правильности коммутации оборудования на основе машинного обучения с подкреплением.

\section{Заключение}

Успешное внедрение виртуальной компьютерной лаборатории в учебный процесс Института системного анализа и управления Государственного университета «Дубна» делает возможным превращение инноваций в жизнь и способствует существенному отрыву вперёд от традиционных образовательных подходов. 


\section{Список литературы}

1. Уразаева Л.Ю. Перспективы виртуальных компьютерных лабораторий // Информационные системы и технологии в моделировании и управлении. Материалы всероссийской научнопрактической конференции. - 2017. - C. 381-385. - [Электронный ресурc]. URL: http://istmu.csrae.ru/ru/2/page/rinc2017.

2. Белов М.А., Лупанов П.Е., Минзов А.С., Токарева Н.А. Система управления виртуальной инфраструктурой на основе визуальных моделей в среде виртуальной компьютерной лаборатории // Современная наука: актуальные проблемы теории и практики. Серия: Естественные и технические науки. - 2019. - № 6-2. - С. 41-46.

3. Belov M.A., Mikheev M.A., Lupanov P.E., Tokareva N.A., Cheremisina E.N., Krukov Y.A. Essential aspects of it training technology for processing, storage and data mining using the Virtual Computer Lab // B сборнике: CEUR Workshop Proceedings. 8. Cep. "GRID 2018 - Selected Papers of the 8th International Conference "Distributed Computing and Grid-Technologies in Science and Education" 2018. - C. 207-212.

4. Belov M.A., Cheremisina E.N., Tokareva N.A. Применение виртуальной компьютерной лаборатории при подготовке ИТ специалистов в области хранения, обработки и интеллектуального анализа больших данных // В сборнике: Преподавание информационных технологий в Российской Федерации. Материалы Шестнадцатой открытой Всероссийской конференции. - 2018. — C. 42-44.

5. Белов М.А., Крюков Ю.А., Лупанов П.Е., Михеев М.А., Черемисина Е.Н. концепция когнитивного взаимодействия с виртуальной компьютерной лабораторией на основе визуальных моделей и экспертной системы // Современная наука: актуальные проблемы теории и практики. Серия: Естественные и технические науки. - 2018. — № 10. - С. 27-35.

6. Белов М.А., Крюков Ю.А., Михеев М.А., Лупанов П.Е., Токарева Н.А., Черемисина Е.Н. Повышение продуктивности освоения распределённых информационных систем в виртуальной компьютерной лаборатории на основе применения технологий контейнеризации и оркестровки контейнеров // Современные информационные технологии и ИТ-образование. — 2018. - Т. 14. № 4. - С. 823-832.

7. Cheremisina E.N., Belov M.A., Tokareva N.A., Nabiullin A.K., Grishko S.I., Sorokin A.V. Embedding of containerization technology in the core of the virtual computing lab // B cборнике: CEUR Workshop Proceedings. 26. Cep. "Selected Papers of the 26th International Symposium on Nuclear Electronics and Computing, NEC 2017" — 2017. - C. 299-302.

8. Елеферов Д.С., Белов М.А. Технология управления жизненным циклом программного обеспечения для проведения орбитальных испытаний спутника связи // Системный анализ в науке и образовании. - 2013. - № 2. - С. 38-46.

9. Серёгин М.С. Использование платформы Arduino в образовательной деятельности // Инновационная наука. - 2019. - № 6. - C. 62-64. - [Электронный pecypc]. URL: https://aeternaufa.ru/journal-innovative-science/\#.

10. Искандаров Р.Н., Шубина Н.В. Проектирование системы контроля и управления доступом на базе микроконтроллера Arduino // Научное обозрение. Педагогические науки. - 2019. — № 3-2. - C. 18-23. - [Электронный pecypc]. URL: https://science-pedagogy.ru/.

11. Семенов Н.Е. Организация взаимодействия между устройствами IоT на базе платформы Arduino \ ESP // Производственные технологии будущего: от создания к внедрению. Материалы Международной научной-практической конференции. — 2019. — С. 265-267. — [Электронный ресурс]. URL: https://www.elibrary.ru/item.asp?id=42330819.

12. Мошков В.В., Андреев Р.А., Прасолов А.А. Разработка программно-аппаратного комплекса для проведения измерений параметров сети мобильной связи на базе Arduino MEGA // Экономика и качество систем связи. - 2019. - №4 (14). — C. 53-61. - [Электронный ресурс]. URL: http://nirit.org/. 